\title{
Simulated Annealing coupled with the Tabu Search Method for Continuum Optimization in Electromagnetics
}

\author{
J. A. Vasconcelos \\ CPDEE - UFMG \\ Av. Antônio Carlos 6627 - 31270-901 - B. Hte. - MG - Brazil \\ L. Krähenbühl \\ A. Nicolas \\ Centre de Génie Electrique de Lyon - ECL. \\ BP163 - 69131 Ecully Cedex - France
}

\begin{abstract}
In this paper the simulated annealing algorithm coupled with the tabu search method for continuum optimization problems is presented. Basically, the new algorithm is obtained by assembling certain characteristics of the tabu search method into the standard simulated annealing. The numerical performance of the new algorithm is discussed based on the optimization of some classical test functions. Finally, an insulator geometry is optimized using this algorithm coupled with the boundary element method in order to test it in electromagnetics.
\end{abstract}

\section{INTRODUCTION}

The simulated annealing algorithm (SA) was first proposed in the area of combinatorial optimization, that is, when the cost function is defined in a discrete domain [1]. In this case, the design variables are discrete and the random moves correspond to permutations in the list of possible moves. It happens as in the traveling salesman problem, where the random moves correspond to permutations in the list of cities to be visited. Therefore, many important problems are defined as functions of continuous variables and the application of the SA requires an efficient strategy to choose the random step $h$. This strategy must be problem independent and self-regulatory to allow SA algorithm to be implemented efficiently and reliably.

Some authors, e.g., Vanderbilt and Louie [2] and Corana et al. [3] have proposed strategies to deal with this problem. The former chose the step as

$$
h=Q u
$$

where the matrix $Q$ controls the step distribution and $u$ is a vector of random numbers $\left(u_{1}, u_{2}, \ldots ., u_{n}\right)$. Each $u_{i}$ is chosen independently. This matrix can be generated as an isotropic distribution with an RMS average step length. The strategy suggested by Corana et al to adjust the step vector is based on the ratio of accepted to rejected moves. This idea consists in changing the step to keep the ratio close to one. A lower rate means that many moves are rejected and the search was made in a too large region, thus misusing computational

Manuscript received July 10, 1995.

J.A. Vasconcelos (joao@cpdee.ufmg.br); L. Krähenbül (krahenb@trotek.ec1.fr); A. Nicolas (alain@trotek.ec-l.fr)

This work was supported in part by the Conselho Nacional de Pesquisa Cientifica e Tecnologica $(\mathrm{CNPq})$ and the Fundação de Amparo à Pesquisa do Estado de Minas Gerais (FAPEMIG) - Brazil. effort. On the other hand, a higher rate means that many moves are accepted and the search was made in a region too small also wasting computational time. In other words, this strategy searches to obtain a state of quasi-equilibrium for each temperature in analogy with the physical process of annealing. They proposed two equations to update the step vector $h$ as given bellow:

$$
\begin{array}{ll}
h_{i}^{\prime}=h_{i}\left[1+c_{i}\left(p_{i}-0.6\right) / 0.4\right] & \text { if } p_{i}>.6 \\
h_{i}{ }^{\prime}=h_{i}\left[1+c_{i}\left(0.4-p_{i}\right) / 0.4\right]^{-1} & \text { if } p_{i}<.4
\end{array}
$$

where $p_{i}$ is the ratio of the number of accepted moves to the number of proposed ones and $c_{i}$ is a control parameter for the $\mathrm{i}$-th direction. Using the equations above, a selfregulatory strategy is obtained. In the initial process, Corana et al. used the value of $h_{i}=0.5\left(b_{i}-a_{i}\right)$, where $a_{i}$ and $b_{i}$ are the inferior and superior bounds for the i-th step.

In the solution of some analytical problems using this last strategy, it is remarked that the results obtained presented some dependency from the starting temperature. The principal cause is due to the difficulty to adjust the step length $h$.

This paper examines how the characteristics of the tabu search algorithm assembled into the standard simulated annealing improves it. The principal idea is to deal with a vector of different step lengths in place of only one step.

\section{TABU SEARCH AlgORITHM}

The Tabu Search method (TS), initially proposed for combinatorial optimization by Glover, was extended to continuous variables by $\mathrm{Hu}$ [4]. Glover's idea, as summarized by $\mathrm{Hu}$, is as follows:

- For the given point $x$ and the given step $h$, let $\mathrm{N}(x, h)$ be the neighbour of $x$, defined as $\mathrm{N}(x, h)=\{x:|x-y| \leq h\}$.

- For the step $h$ and the direction $r$, let $y$ be the move from $x$, i.e., $y=x+r h$, where $r$ is a random number that belongs to $(-1,1)$.

- Given the set $\mathrm{H}=\left\{h_{1}, h_{2}, \ldots, h_{n d}\right\}$, the list of inactive steps $\mathrm{T}$, which is initially empty, and an initial feasible point $x$, the search of the next points are made over a set of active neighbours $\mathrm{N}\left(x, h_{k}\right)$, where $k$ belongs to $\mathrm{H}-\mathrm{T}$. Only one feasible random move is generated for each neighbour. If a decrement in the objective function is obtained for $y$, 
then save $y$ as the current solution $x$ and add its corresponding step $h$ to T. When T is full, T is updated empty and the total process is reiterated, otherwise the above procedure is repeated for the next active step.

In global optimization problems, the moves in neighbours of different sizes prevent the search from trapping at a local optimum.

\section{THE METHOD}

The flow chart of the modified simulated annealing algorithm (MSA) is given bellow. Basically, it is the SA algorithm proposed by Corana et al (as applied by Simkin \& Trowbridge [5]) with some characteristics of the TS algorithm incorporated [6].

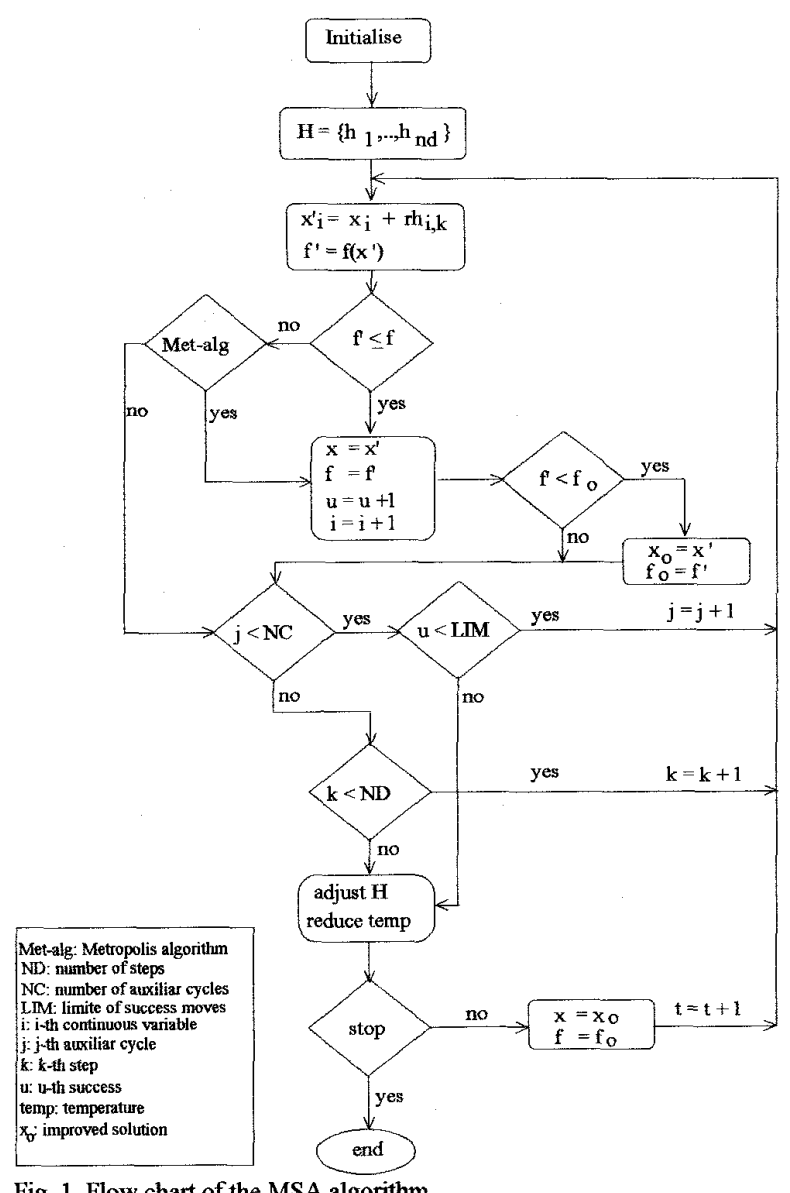

Fig. 1. Flow chart of the MSA algorithm.

The main difference between the MSA proposed here and the SA is that in the former the vector of steps $h$ is replaced by $N D$ vectors of steps, which are stored in matrix $H$. Also, $N C$ auxiliary cycles are executed for each active vector $h_{k} k$ $\in\{1, \ldots, N D\}$. After $N C^{*} N D$ cycles or if $\mathbf{u}>\mathrm{LIM}$, matrix $H$ is adjusted, using the same criteria as in [3] and the temperature in the SA is reduced by a factor smaller but close to 1 .
The MSA starts with a given initial point and a succession of them is generated by changing the value of one variable at time with the rule $x_{i}^{\prime}=x_{i}+r h_{i, k}$. In this equation $i$ denotes the $i$-th variable, $k$ the $k$-th step and $r$ is a number whose values could be randomly $+l$ or -1 . The last suggestion is proposed in [7].

The procedure used to calculate matrix $H$ [4], is:

- First, consider that the objective function is defined as a function of $n$ continuous variables $x_{i}, i \in\{1, \ldots, n\}$, and each $x_{i} \in\left\{a_{i}, b_{j}\right\}$.

- Now, the $N D$ vectors of $H$ can be calculated as follows:

$$
\begin{gathered}
h_{i, 1}=\left(b_{i}-a_{j}\right) / c \\
h_{i, 2}=h_{i, 1} / c \\
\vdots \\
h_{i, n d}=h_{i, n d-1} / c
\end{gathered}
$$

where $c$ is a factor greater than 1 , for example $c=2$

The condition when the number of success moves $u$ is less than $L I M$ in the MSA is used as a supplementary test. It enables the algorithm to update matrix $\mathrm{H}$ and the temperature TEMP, when the number of success moves reaches the limit imposed. This condition save computational time because of the total number of auxiliary cycles that is not completely iterated.

The deviation between two or more consecutive values of the objective function, in addition to the test if the value of the temperature is less than a small number, say TOLTEMP, and a limit in the number of function evaluations can be used as a stop criteria.

\section{COUPLING THE MSA WITH A CODE OF ELECTROMAGNETIC Field Calculation}

A code based on the finite element method or boundary element method can be coupled with the MSA algorithm via the objective function. The constrained non-linear optimization problem in electromagnetics generally involves problems of the form:

$$
\begin{array}{ll}
\min & f_{l}(x) \\
\text { with } & g_{l}(x) \leq 0 \quad l=1, \ldots, m
\end{array}
$$

where $f_{l}(x)$ is the objective function, $g_{l}(x)$ is the 1-th constraint function and $x=\left(x_{1}, x_{2}, \ldots x_{n}\right)$ is the vector of $n$ degrees of freedom ( $x$ can be either a vector of geometric parameters and/or electrical field or other electrical quantity that can be implicitly function of the geometric parameters).

Using a transformation as in the penalty methods, the original problem stated above can be rewritten as an unconstrained minimization problem, as shown in (4).

$$
\min f(x)=f_{l}(x)+p \sum_{l=1}^{m}\left[g_{l}(x)\right]
$$

Above, $p$ is the penalty parameter and the term $\left[g_{l}(x)\right]_{+}$ ensures that only the violated constraint function is 
considered. Now, the transformed problem can be solved by the MSA algorithm.

\section{RESULTS}

Analytical Results

A computer code which was developed based on the MSA algorithm was tested using the same two test functions reported in [8]. They are the "exponential" function $f_{1}$ and the Rastrigin's function $f_{2}$.

The function $f_{1}$ is a continuous, bimodal function of two variables $x=\left(x_{1}, x_{2}\right)^{T}$.

$$
\begin{gathered}
f_{1}(x)=20-e^{\left(-\sum\left(x_{i}-1.5\right)^{2}+1\right)}+e^{\left(-\sum\left(x_{i}-2.5\right)^{2}+1.05\right)} \\
-e^{\left(-\sum\left(x_{i}-3.5\right)^{2}+1.1\right)} \\
0 \leq x_{i} \leq 10 \quad i=1,2
\end{gathered}
$$

This function has two local minima. The global minimum can be found at $x^{*}=(3.59585 ; 3.59585)^{T}$ to give $f_{1}\left(x^{*}\right)=17.30889$.
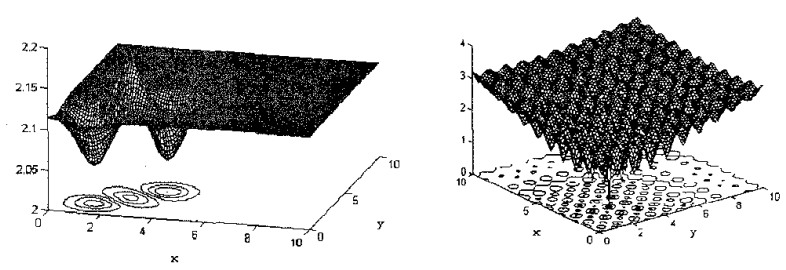

Fig. 2 (a)."Exponential" function $\mathrm{f}_{1}(\mathrm{x})$ and (b) Rastrigin's function $\mathrm{f}_{2}(\mathrm{x})$

The second function $f_{2}$ is a continuous, multimodal test function of $n$ continuous variables. For $x_{i} \in\{0,10\}$ there are $10^{\mathrm{n}}$ local minima and the global minimum can be found at $x^{*}=(2.5 ; \ldots ; 2.5)^{T}$ giving $f_{2}\left(x^{*}\right)=0$.

$$
\begin{gathered}
f_{2}(x)=10 n+\sum_{i=1}^{n}\left[\left(x_{i}-2.5\right)^{2}-10 \cos 2 \pi\left(x_{i}-2.5\right)\right] \\
n=10 \quad x=\left(x_{1}, \ldots, x_{10}\right)^{T} \quad 0 \leq x_{i} \leq 10
\end{gathered}
$$

Several computation using different start points and ND values were executed. The start points $A, B, C$ and $D$ for the first function and $A^{\prime}, B^{\prime}, C^{\prime}$ and $D^{\prime}$ for the second one were used:

$$
\begin{aligned}
& \mathrm{A}=\{1.0,9.0\}^{\mathrm{T}} \\
& \mathrm{B}=\{0.0,1.0\}^{\mathrm{T}} \\
& \mathrm{C}=\{4.0,1.0\}^{\mathrm{T}} \\
& \mathrm{D}=\{7.0,9.0\}^{\mathrm{T}} \\
& \mathrm{A}^{\prime}=\{0.5,0.2,0.3,0.4,5 \cdot 0,9.0,8 \cdot 2,2 \cdot 0,4 \cdot 0,3.2\}^{\mathrm{T}} \\
& \mathrm{B}^{\prime}=\{1.0,2.0,3 \cdot 0,4.0,5 \cdot 0,6.0,7.0,8 \cdot 0,9.0,10.0\}^{\mathrm{T}} \\
& \mathrm{C}^{\prime}=\{1.0,1.0,1.0,1.0,1.0,1.0,1.0,1.0,1.0,1.0\}^{\mathrm{T}} \\
& \mathrm{D}^{\prime}=\{0.0,10.0,0.0,10.0,0.0,10.0,0.0,10.0,0.0,10.0\}^{\mathrm{T}}
\end{aligned}
$$

The termination criteria used was only the test on the temperature value. The main parameters used were: $\mathrm{ND}=\{1,2, . ., 20\}, \quad \mathrm{NC}=10^{*} \mathrm{n} / \mathrm{ND}, \quad \mathrm{LIM}=\mathrm{MIN}(\mathrm{n}, \mathrm{ND})$,

\begin{tabular}{|c|c|c|c|c|c|c|}
\hline$\overline{\text { Point }}$ & ND & 1 & 5 & 10 & 15 & 20 \\
\hline \multirow[t]{2}{*}{$\bar{A}$} & $\mathrm{NBC}$ & 909 & 1102 & 855 & 751 & 842 \\
\hline & $\mathrm{q}_{1}^{*}$ & 0.18334 & 0.00037 & 0.00003 & 0.00014 & 0.00006 \\
\hline \multirow[t]{2}{*}{$\bar{B}$} & $\mathrm{NBC}$ & 811 & 1097 & $832\left(^{*}\right)$ & 724 & 724 \\
\hline & $\mathrm{q}_{1}^{*}$ & 0.03072 & 0.00076 & 0.28051 & 0.00033 & 0.00033 \\
\hline \multirow[t]{2}{*}{$\overline{\mathrm{C}}$} & $\mathrm{NBC}$ & 712 & 1091 & 921 & 763 & 834 \\
\hline & $\mathrm{q}_{1}{ }^{*}$ & 0.07934 & 0.00059 & 0.00017 & 0.00003 & 0.00011 \\
\hline \multirow[t]{2}{*}{$\overline{\mathrm{D}}$} & NBC & 758 & 1056 & 880 & 763 & 777 \\
\hline & $\mathrm{q}_{1}{ }^{*}$ & 0.49174 & 0.00026 & 0.00016 & 0.00019 & 0.00011 \\
\hline
\end{tabular}
TEMP $^{0}=0.1 \quad$ ("Exponential" function), TEMP $^{0}=1.0$
(Rastrigin's function), TEMP $^{\mathrm{t}+1}=0.95 * \mathrm{TEMP}^{\mathrm{t}}$, TOLTEMP $=0.001$ and $\mathrm{C}=2$.

The results are shown in TABLE $\mathrm{I}$ and $\mathrm{II}$, where $q_{1}^{*}=f_{1}\left(x_{\partial}\right)-f_{1}\left(x^{*}\right)$ and $q_{2}{ }^{*}=f_{2}\left(x_{\partial}\right)$. The number of function evaluations is denoted by NBC. Note that the total number of possible cycles at each temperature $N C^{*} N D$ were made constant and equal to $10^{*} n$, where $n$ is the number of design variables. These computation were performed using simple precision.

TABLE I

"EXPONENTLAL" FUNCTION F

TABLE II

\begin{tabular}{lrrrrrr}
\multicolumn{7}{c}{ RASTRIGN's FUNCTION $\mathrm{F}_{2}}$. \\
\hline Point & $\mathrm{ND}$ & 1 & 5 & 10 & 15 & 20 \\
\hline $\mathrm{A}^{\prime}$ & $\mathrm{NBC}$ & $4825\left(^{*}\right)$ & 7738 & 7534 & 6311 & 5504 \\
& $\mathrm{q}_{2}{ }^{*}$ & 18.07629 & 0.32427 & 0.00920 & 0.00468 & 0.00539 \\
\hline $\mathrm{B}^{\prime}$ & $\mathrm{NBC}$ & $3345\left(^{*}\right)$ & 7145 & 7453 & 5919 & 5450 \\
& $\mathrm{q}_{2}{ }^{*}$ & 10.52338 & 0.54167 & 0.00784 & 0.00360 & 0.00636 \\
\hline $\mathrm{C}^{\prime}$ & $\mathrm{NBC}$ & $4679\left(^{*}\right)$ & 8145 & 7864 & 6242 & 5692 \\
& $\mathrm{q}_{2}{ }^{*}$ & 5.40686 & 0.20988 & 0.01038 & 0.00281 & 0.00237 \\
\hline $\mathrm{D}^{\prime}$ & $\mathrm{NBC}^{2}$ & $2166\left(^{*}\right)$ & 7382 & 7219 & 5617 & 5282 \\
& $\mathrm{q}_{2}{ }^{*}$ & 10.52417 & 0.00000 & 0.01090 & 0.00634 & 0.00000 \\
\hline
\end{tabular}

The performance comparison in terms of accuracy and the number of function evaluations, for several values of ND, using three points generated randomly, is presented in Fig. 3. It was used the same parameter values as before except for TOLTEMP, which it was made equal to $1 \mathrm{E}-5$.

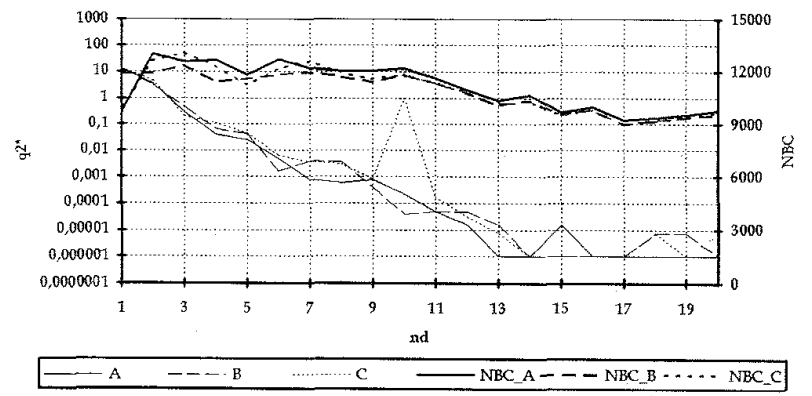

Fig. 3. Rastrigin's function $f_{2}(x)$ with ten variables generated randomly.

The MSA works quite well for all $N D$ values. It has been noted that the best results are given by using a number of steps greater than ten $(N D>10)$. For $N D=1$, the algorithm works as the standard SA. It is interesting to point out that for $N D=1$, the number of function evaluations necessary to reach the optimal result is large, and the results are dependent upon the starting temperature in the SA. Changing the test $u<L L M$ in the MSA to $\mathrm{u}<5 * \mathrm{LIM}$, after 
to $\mathrm{u}<10^{*} \mathrm{LIM}$ and $\mathrm{u}<100^{*} \mathrm{LIM}$, for the point $\mathrm{A}^{\prime}$, the standard SA reaches a point near the global minimum only for the last case. In this test, the number of field evaluations was 8224,10380 and 17218 respectively. The values of the objective function in this three cases were $4.28085,8.38059$ and 2.09673 in the same order.

\section{Electrostatic Insulator}

The goal in this problem is to find a maximum normal electrical field on the lower electrode equal to $E_{0}$. The optimization problem was formulated in the following way:

$$
\begin{array}{ll}
\min & f(x)=\left(E_{\max }^{2}-E_{0}^{2}\right)^{2} \\
\text { with } \quad x_{\text {min }_{i}} \leq x_{i} \leq x_{\max _{i}} \quad i=1, \ldots, 5
\end{array}
$$

where $x$ is the vector of design variables (Fig. 4). $E_{\max }$ and $E_{o}\left(E_{O}=130 \mathrm{~V} / \mathrm{m}\right)$ are respectively the maximum calculated and the desired normal electric fields on the movable boundary.

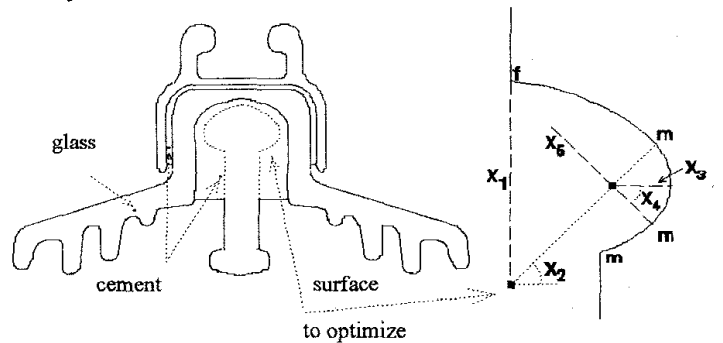

Fig. 4. Insulator for distribution lines (left) and a zoom of the boundary to be optimized showing the design parameters.

Two optimization are presented for different values of $N D$, in order to show the power and the applicability of the new algorithm. The parameter values used in the MSA to solve this problem were: $N D=\{10,20\}, N C=16^{*} n / N D, L M$ $=8^{*}, T^{*} M^{t+1}=0.95^{*} T E M P^{t}, T^{t} M^{0}=0.1$ and $C=2$.

The termination criterion used in this case was simply the test on the number of function evaluations, after each cycle of temperature. This choice was made to prevent a great number of field analysis. Using this test, the number of cycles of temperature performed were only one for the first case and two for the second one.

The results are shown in TABLE III, where the optimized parameters for $N D=10$ and $\mathrm{ND}=20$ are denoted by Xopt $\mathrm{X}_{10}$ and $\mathrm{Xopt}_{20}$, respectively. The maximum normal electric field calculated before the optimization was $188.61 \mathrm{~V} / \mathrm{m}$.

The optimized parameters for the two simulation cases are approximately equals. Note that the parameter $x_{3}$ reaches its maximum value in both the cases. Also, these results show that MSA can be used to optimize real problems.

Fig. 5 shows the normal electric fields for the initial and the optimized geometries for the different optimizations as functions of the ordinate $\mathrm{z}$ along the movable boundary.

The result obtained represents a considerable gain on the maximum normal electric field, which can be considered a satisfactory result.
TABLE III

\begin{tabular}{|c|c|c|c|c|c|}
\hline Parameters & Xinit & Xmin & $X \max$ & Xopt $_{10}$ & Xopt $_{20}$ \\
\hline radius $x_{1}(m)$ & .019 & .010 & .025 & .01736 & .01719 \\
\hline angle $x_{2}$ & 48.0 & 40 & 60 & 54.88 & 54.73 \\
\hline radius $x_{3}(m)$ & .0025 & .002 & .010 & .010 & .010 \\
\hline angle $x_{4}$ & -10.0 & -20 & 0 & -18.11 & -18.69 \\
\hline radius $x_{5}(\mathrm{~m})$ & .019 & .010 & .025 & .014 & .01402 \\
\hline $\operatorname{Emax}(\mathrm{V} / \mathrm{m})$ & 188.61 & (2) & 2. & 132.99 & 132.97 \\
\hline NBC & 898 & & 40" & 80 & 138 \\
\hline
\end{tabular}

INTIAL AND FINAL DESIGN VARIABLE VALUE FOR ND $=\{10,20\}$.

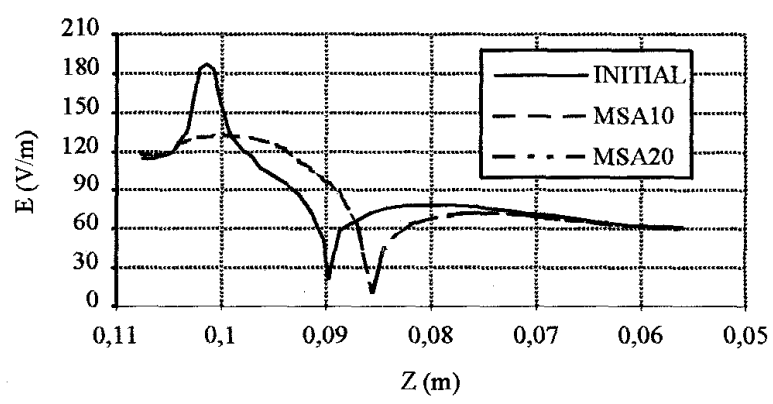

Fig. 5. Normal electric field on the movable boundary for the initial and the optimized geometries $(N D=\{10,20\})$

\section{CONCLUSION}

A new simulated annealing algorithm coupled with the tabu search method for design optimization was presented in this paper. The analytical results have shown that MSA can be applied to solve complex global optimization problems. Among the advantages of MSA, it can be mentioned that no calculation of gradient is necessary and also that its implementation is very simple. When solving a real problem, if the main interest is to find an improved solution, the number of function evaluations can be used to prevent numerous electromagnetic field computation. Moreover, a small number of auxiliary cycles in addition to a small TEMP value will also work in the same way.

\section{REFERENCE}

[1] S. Kirkpatrick, C.D. Gelatt, and M.P. Vecchi, "Optimization by simulated annealing", Science, 220,671-680, 1983.

[2] D. Vanderbilt and S.G. Louie, "A Monte Carlo simulated annealing approach to optimization over continuous variables", Journal of Computational Physics, 56, 259-271, 1984.

[3] A. Corana, M. Marchesi, C. Martini and S. Ridella, "Minimizing multimodal functions of continuous variables with the 'Simulate Annealing' algorithm", ACM Trans. on Mathematical Software, 13, 262$280,1987$.

[4] N. Hu, "TABU search method with random moves for globally optimal design", Int. J: Num. Meth. Engineering, 35, 1055-1070, 1992.

[5] J. Simkin and C.W. Trowbridge, "Optimizing electromagnetic devices combining direct search methods with simulated annealing", IEEE Trans. on Magnetics, 28, 1545-1548, 1992.

[6] J.A. Vasconcelos, "Optimisation de forme des structures électromagnetics", Doctoral Thesis, ECL 94-32, France.

[7] G. Drago, A. Manella, M. Nervi, M. Repetto, G. Secondo, "A combined strategy for optimization in non-linear magnetic problems using simulated annealing and search techniques", IEEE Trans. on Magnetics, $28,1541-1544,1992$.

[8] J.A. Vasconcelos, L Krähenbühl, L Nicolas, A Nicolas, "Design optimisation using the BEM coupled with Genetic Algorithm", Proc. of the 2nd IEE Inter. Conference on Comp. in Electromagnetics, Nottinghan (UK), 1994 